improve quality of care, improve adherence to BTS guidelines, reduce in-hospital mortality and prevent ITU admission.

We believe the guideline and protocol supported the on-call teams to identify and safely manage respiratory failure. We continue to evaluate the service.

\section{P193 EARLY NON-INVASIVE VENTILATION VERSUS STANDARD OXYGEN THERAPY IN IMMUNOCOMPROMISED PATIENTS WITH RESPIRATORY FAILURE: A META-ANALYSIS}

RV Villalobos, UK Gopez, K Flores. Department of Medicine, Philippine General Hospital, Manila, Philippines

\subsection{6/thoraxjnl-2016-209333.336}

Background and aims Respiratory failure is common in immunocompromised patients. Intubation and mechanical ventilation (MV) is the mainstay of treatment but is associated with increased risk of pneumonia and other complications. Non-invasive ventilation (NIV) is an alternative to MV in a select group of patients and aims to avoid the complications of MV.

In these patients, we performed a meta-analysis on the effect of early NIV versus conventional oxygen therapy in reducing intubation rates and other important clinical outcomes.

Methods We performed an extensive online and unpublished data search for relevant studies that met the inclusion criteria. We included randomised controlled trials that used early NIV versus conventional oxygen therapy in immunocompromised patients with respiratory failure. Risk of bias and acceptability assessment were independently performed by the authors.

The primary outcome of interest was intubation and MV rate. The secondary outcomes were ICU and all-cause mortality, ICU length of stay and duration of mechanical ventilation.

Results Four studies with a total of 553 patients met the criteria for inclusion and were included in the analysis.

Patients given NIV were $38 \%$ less likely to be intubated vs. those given oxygen, RR 0.62 (95\% CI: 0.42, 0.93); however, this result is significantly heterogenous. After sensitivity analysis, results showed $48 \%$ less likelihood of intubation and mechanical ventilation in the group treated with NIV, RR 0.52 (95\% CI: $0.35,0.77)$.

Patients on NIV had 1.18days less stay in the ICU vs. oxygen group (95\% CI: $-1.84,-0.5$ days). There was no statistically significant decrease in all-cause mortality between the two groups, RR 0.84 (95\% CI: 0.63, 1.13), but this effect is heterogenous. After another sensitivity analysis performed specifically for this outcome, results showed a $25 \%$ significant reduction in all cause mortality in patients given NIV vs. oxygen therapy, RR 0.75 (95\% CI: 0.58, 0.96).

There is no difference in the duration of mechanical ventilation between groups.

Conclusions In immunocompromised patients with respiratory failure, early NIV reduced intubation rates and decreased allcause mortality and length of ICU stay compared to standard oxygen therapy.

\section{P194 EARLY WARNING SCORES, TOO IMPRECISE A TOOL IN PATIENTS WITH RESPIRATORY DISEASE?}

${ }^{1} \mathrm{~S}$ Forster, ${ }^{2} \mathrm{G}$ Housley, ${ }^{2} \mathrm{~J}$ Hatton, ${ }^{1} \mathrm{D}$ Shaw. ${ }^{1}$ University Of Nottingham, Nottingham, UK; ${ }^{2}$ Nottingham University Hospitals Trust, Nottingham, UK

\subsection{6/thoraxjnl-2016-209333.337}

Introduction Guidance from the National Institute of Health and Care Excellence in $2007^{1}$ has led to the almost universal use of early warning scores (EWS) derived from vital signs observations in hospitals in the UK to highlight patients at risk of deterioration. Lack of high quality prospective studies limits our understanding of the impact of using such monitoring systems on outcomes and working patterns. No EWS has been validated in respiratory patients despite widespread use. Our aim was to examine the ability of both the locally used EWS and National Early Warning Score (NEWS) to predict patient deterioration and associated burden of escalations generated in a respiratory cohort.

Methods Vital signs observations and outcomes for all admissions under the respiratory department at a tertiary referral centre between April 2015 and March 2016 were analysed. Predicted and actual escalation patterns in relation to primary endpoint of mortality were examined comparing NEWS to local EWS. Patients documented as receiving end of life care were removed from analysis.

Results Over 12 months there were 165,184 observations sets during 5293 admissions, with a mean of 38 observations per admission (standard deviation 50). Occurrence of primary endpoint of in-hospital death was $6.74 \% .13 \%$ of observations triggered clinical escalation to a registered nurse or beyond, with mean of 1075 per month. 112 (31\%) patients who died did not trigger escalation on their final set of observations, 1 patient was escalated despite scoring below protocol threshold. Applying NEWS criteria retrospectively predicts 6 patients who died would not be escalated, while generating a mean of 12,409 escalations of vital signs observations per month to registered nurse or beyond, 1,621 in patients who went on to die in hospital.

\begin{tabular}{|c|c|c|c|c|c|c|c|c|c|c|}
\hline Study or Subgroup & \multicolumn{2}{|l|}{ NIV } & \multicolumn{2}{|c|}{ Oxygen Alone } & Weight & $\begin{array}{c}\text { Risk Ratio } \\
\text { M-H, Random, } 95 \% \mathrm{Cl}\end{array}$ & \multicolumn{4}{|c|}{$\begin{array}{c}\text { Risk Ratio } \\
\text { M-H, Random, } 95 \% \mathrm{Cl}\end{array}$} \\
\hline Antonelli 2000 & 4 & 20 & 14 & 20 & $13.8 \%$ & $0.29[0.11,0.72]$ & & $\longrightarrow$ & & \\
\hline Hilbert 2001 & 12 & 26 & 20 & 26 & $29.9 \%$ & $0.60[0.38,0.96]$ & & & & \\
\hline Lemiale 2015 & 73 & 191 & 82 & 183 & $42.1 \%$ & $0.85[0.67,1.09]$ & & & & \\
\hline Wermke 2012 & 6 & 43 & 11 & 44 & $14.2 \%$ & $0.56[0.23,1.37]$ & & & & \\
\hline Total $(95 \% \mathrm{Cl})$ & & 280 & & 273 & $100.0 \%$ & $0.62[0.42,0.93]$ & & & & \\
\hline Total events & 95 & & 127 & & & & & & & \\
\hline $\begin{array}{l}\text { Heterogeneity: Tau² } \\
\text { Test for overall effect }\end{array}$ & $\begin{array}{l}0.09 ; \mathrm{Chi} \\
Z=2.31\end{array}$ & $\begin{array}{l}=6.6 \\
P=0.0\end{array}$ & $\begin{array}{l}0, d f=3(f \\
2)\end{array}$ & 0.09); & $I^{2}=55 \%$ & & $\stackrel{\vdash}{\circ .01}$ & $\begin{array}{c}0.1 \\
\text { Favours NIV }\end{array}$ & $\begin{array}{c}10 \\
\text { Favours Oxygen }\end{array}$ & 100 \\
\hline
\end{tabular}

